\title{
Adapting fuzzy rough sets for classification with missing values
}

\author{
Oliver Urs Lenz ${ }^{10000-0001-9925-9482]}$, Daniel Peralta ${ }^{1,2}$ [0000-0002-7544-8411], \\ and Chris Cornelis ${ }^{1}[0000-0002-7854-6025]$ \\ 1 Computational Web Intelligence, Department of Applied Mathematics, Computer \\ Science and Statistics, Ghent University \{oliver. lenz, chris.cornelis\}@ugent. be \\ http://www.cwi.ugent.be \\ 2 Data Mining and Modelling for Biomedicine group, VIB Center for Inflammation \\ Research, Ghent University daniel. peralta@irc.vib-ugent.be \\ https://www.irc.ugent.be
}

\begin{abstract}
We propose an adaptation of fuzzy rough sets to model concepts in datasets with missing values. Upper and lower approximations are replaced by interval-valued fuzzy sets that express the uncertainty caused by incomplete information. Each of these interval-valued fuzzy sets is delineated by a pair of optimistic and pessimistic approximations. We show how this can be used to adapt Fuzzy Rough Nearest Neighbour (FRNN) classification to datasets with missing values. In a small experiment with real-world data, our proposal outperforms simple imputation with the mean and mode on datasets with a low missing value rate.
\end{abstract}

Keywords: Fuzzy rough sets · Interval-valued fuzzy sets · Machine learning $\cdot$ Missing values

\section{Introduction}

Fuzzy and rough sets can be used to model different types of uncertainty. Fuzzy sets [3,23] allow us to model partial membership of a concept, while rough sets $[20,21]$ capture the conflicting ways in which a concept may be predicted from a set of independent attributes. The two concepts are unified in the fuzzy rough set [8]. If $X \subset \mathbb{R}^{m}$ is a dataset, and $C \subseteq X$ a fuzzy subset, then the fuzzy rough set induced by $C$ is the pair of fuzzy sets $(\bar{C}, \underline{C})$. The upper approximation $\bar{C}$ generalises the positive evidence for $C$ in $X$, whereas the lower approximation $\underline{C}$ generalises the negative evidence for $C$ in $X$.

In this paper, we consider a third type of uncertainty: incomplete information. There exists a wide range of strategies to deal with missing data [2], including proposals that involve rough or fuzzy rough sets [22]. In particular, fuzzy rough sets have been used for imputation [1], there have been proposals to adapt both crisp and fuzzy decision rules to the presence of missing values $[10,12,17]$, and in the context of classical rough sets, [10] has provided three alternative definitions of upper and lower approximations in datasets with missing values. In contrast, our strategy is to incorporate the uncertainty of incomplete information directly into the representation of concepts, by extending the notion of fuzzy rough set. 
We propose to mimic the dual approach of rough sets by modelling an optimistic and a pessimistic scenario when comparing a missing value with another value. The optimistic scenario is that the two values are really identical, while the pessimistic scenario is that they are maximally different. We cannot know what the ground truth is, but we know that it must lie somewhere in between these two extremes. Formally, we can represent this with an interval-valued fuzzy set [9, and references therein]. Since the uncertainty of incomplete information is orthogonal to the uncertainty that arises from positive and negative information, the resulting interval-valued fuzzy rough set is defined by four fuzzy sets: the optimistic and pessimistic upper and lower approximations $\bar{C}^{\min }, \bar{C}^{\max }, \underline{C}_{\min }, \underline{C}_{\max }$.

This work builds on the earlier proposal for interval-valued fuzzy rough sets in the context of feature selection [14], as well as a related proposal of ill-known fuzzy rough sets [5] based on twofold fuzzy sets [7], but this approach has otherwise remained relatively underexplored. We present an up-to-date definition in Section 2. In Section 3, we modify Fuzzy Rough Nearest Neighbour (FRNN) classification to incorporate interval-valued fuzzy rough sets, and evaluate its performance on a number of real-live datasets.

\section{Interval-valued fuzzy rough sets}

Recall the formal definitions of the upper and lower approximations with Ordered Weighted Averaging (OWA) operators [4]:

Definition 1 (Soft maxima and minima). Let $w$ be a weight vector of length $k$, with values in $[0,1]$ that sum to 1 . The soft maximum $w \downarrow$ and soft minimum $w \uparrow$ induced by $w$ transform a collection $Y$ of values in $\mathbb{R}$ into, respectively, the weighted sums

$$
\begin{aligned}
& \underset{i \leq k}{w \downarrow} Y=\sum_{i \leq k} w_{i} \cdot y_{i^{+}}, \\
& \underset{i \leq k}{w \uparrow} Y=\sum_{i \leq k} w_{i} \cdot y_{i^{-}},
\end{aligned}
$$

where $y_{i^{+}}$and $y_{i^{-}}$are the $i$ th largest and ith smallest elements in $Y$.

Definition 2 (Upper and lower approximations). Let $X \subset \mathbb{R}^{m}$ be a finite multisubset for some $m \in \mathbb{N}$, let $R$ be a tolerance relation on $\mathbb{R}^{m}$, w a weight vector of some length length $k, T$ a t-norm and $I$ a fuzzy implication. Then for any fuzzy submultiset $C$ of $X$, the upper and lower approximations $\bar{C}$ and $\underline{C}$ are the fuzzy subsets of $\mathbb{R}^{m}$ defined by:

$$
\begin{aligned}
& \bar{C}(y)=\underset{x \in X}{w \downarrow}(T(R(y, x), C(x)) \\
& \underline{C}(y)=\underset{x \in X}{w \uparrow}(I(R(y, x), C(x))
\end{aligned}
$$

While a dataset can contain instances with identical attribute values but different membership degrees in a concept $C$, the upper and lower approximations 
of $C$ only depend on attribute values, and so can be defined as fuzzy subsets of the attribute space.

It is convenient to aggregate $R$ from attribute-specific tolerance relations $R_{i}$ on $\mathbb{R}$ by means of some monotonic function $f:[0,1]^{m} \longrightarrow[0,1]$. In line with recent works, we let $f$ be the mean, and we write $R_{f}:=f\left(\left(R_{i}\right)_{i \leq m}\right)$.

Next, recall the definition of the interval-valued fuzzy set $[9$, and references therein]:

Definition 3 (Interval-valued fuzzy set). Let $X$ be a set. An interval-valued fuzzy set in $X$ is a pair of fuzzy sets $\left(F_{1}, F_{2}\right)$ in $X$ such that $F_{1}(x) \leq F_{2}(x)$ for all $x \in X$.

Equivalently, an interval-valued fuzzy set in $X$ can also be defined as a function $X \longrightarrow \mathcal{I}([0,1])$, where the range is the set of intervals in $[0,1]$, i.e. the subset of $[0,1] \times[0,1]$ whose values in the first component are always less than or equal to the values in the second component.

We can accommodate the possibility of missing data by adjoining a formal symbol denoting a missing value to each copy of $\mathbb{R}$ to obtain $\mathbb{R}_{\text {? }}:=\mathbb{R} \cup\{$ ? $\}$, and by letting $X$ be a multisubset of $\mathbb{R}_{?}^{m}$. The task then is to extend any choice of $R_{i}$ to ?. We define optimistic and pessimistic per-attribute relations $R_{i}^{\max }$ and $R_{i}^{\text {min }}$ by stipulating that for any $a, b \in \mathbb{R}$ :

$$
\begin{array}{lll}
R_{i}^{\max }(a, b)=R_{i}^{\min }(a, b) & =R_{i}(a, b) & \\
R_{i}^{\max }(a, ?)=R_{i}^{\max }(?, b) & =R_{i}^{\max }(?, ?) & =1 \\
R_{i}^{\min }(a, ?)=R_{i}^{\min }(?, b) & =R_{i}^{\min }(?, ?) & =0
\end{array}
$$

Accordingly, we define interval-valued upper and lower approximations through the aggregated relations $R_{f}^{\max }$ and $R_{f}^{\min }$ :

Definition 4 (Interval-valued upper and lower approximations). Let $X \subset \mathbb{R}_{?}^{m}$ be a finite multisubset for some $m \in \mathbb{N}$, let $w$ be a weight vector of some length $k, T$ a t-norm and $I$ a fuzzy implication, and let $\left(R_{i}\right)_{i \leq m}$ be a series of similarity measures and $f: \mathbb{R}^{m} \longrightarrow[0,1]$ an aggregation function such that $R_{f}$ is a tolerance relation. Then for any fuzzy submultiset $C$ of $X$, the intervalvalued upper and lower approximations of $C$ are, respectively, the interval-valued fuzzy sets $\left(\bar{C}^{\min }, \bar{C}^{\max }\right)$ and $\left(\underline{C}_{\min }, \underline{C}_{\max }\right)$, defined as:

$$
\begin{aligned}
& \bar{C}^{\min }(y)=\underset{x \in X}{w \downarrow}\left(T\left(R_{f}^{\min }(y, x), C(x)\right)\right. \\
& \bar{C}^{\max }(y)=\underset{x \in X}{w \downarrow}\left(T\left(R_{f}^{\max }(y, x), C(x)\right)\right. \\
& \underline{C}_{\min }(y)=\underset{x \in X}{w \uparrow}\left(I\left(R_{f}^{\max }(y, x), C(x)\right)\right. \\
& \underline{C}_{\max }(y)=\underset{x \in X}{w \uparrow}\left(I\left(R_{f}^{\min }(y, x), C(x)\right)\right.
\end{aligned}
$$


Because t-norms and fuzzy implications are respectively monotonic and antimonotonic in the first argument, the pessimistic approximations $\bar{C}^{\text {min }}$ and $\underline{C}_{\min }$ encode the minimum membership degrees in the upper and lower approximations, while the optimistic approximations $\bar{C}^{\max }$ and $\underline{C}_{\max }$ encode the maximum membership degrees.

The computational complexity of calculating (4) is in principle the same as that of calculating (2), which is the computational complexity of a $k$-nearest neighbour query. However it requires the implementation of $k$-nearest neighbour algorithms with the distance measure corresponding to (3).

Membership in the optimistic and pessimistic approximations - like membership in ordinary upper and lower approximations - is determined purely on the basis of the attribute values of an instance, so it is possible to plot membership degrees across the attribute space. This is illustrated for a toy example in Fig. 1. Here, $C$ is a crisp set containing two elements, one of which has a missing attribute value, which we have represented with a line. We have chosen $R_{i}(y, x)=1-\left|y_{i}-x_{i}\right|$. For crisp sets, the choice of t-norm becomes void: $T(R(y, x), C(x))$ is equal to $R(y, x)$ if $C(x)=1$, and equal to 0 otherwise. Similarly, the choice of fuzzy implication resolves to a choice of fuzzy negation; we use the standard negation $z \longmapsto 1-z$. We set $w=\left\langle\frac{2}{3}, \frac{1}{3}\right\rangle$. Darker shades of red indicate higher membership degrees. It can be seen that membership degrees of the optimistic approximations are uniformly higher than membership degrees of the pessimistic approximations.

The treatment in this section is essentially an updated version of [14]. The differences are mainly practical. Firstly, [14] uses a more general setting, where $R_{i}$ is an interval-valued relation, but this greater generality potentially obscures the fact that this approach can be applied in any context that currently uses ordinary fuzzy rough sets, where $R_{i}$ is scalar-valued. And secondly, [14] requires the aggregation function $f$ to be a t-norm. As a result, $R_{f}^{\min }$ will always be 0 if any of the attribute values are missing, and we lose the information encoded by the non-missing attribute values.

\section{$3 \quad$ FRNN with interval-valued approximations}

Upper and lower approximations can be used for Fuzzy Rough Nearest Neighbour (FRNN) classification [13], by calculating the membership of an unknown instance in the upper and lower approximations of the crisp decision classes and identifying the class with the highest membership degree. For datasets with missing data, we can instead use the interval-valued upper and lower approximations for classification. We test this with a small experiment. As upper and lower approximations produce equivalent results with two-class datasets, we simplify the experiment by only using the upper approximation.

For crisp sets $C$, the choice of t-norm in (4) becomes void. In line with previous work [18] we use linearly decreasing weights $\frac{k}{k(k+1) / 2}, \frac{k-1}{k(k+1) / 2}, \ldots, \frac{1}{k(k+1) / 2}$, and set $k$ to 20 or the size of the decision class, whichever is smaller. For the 


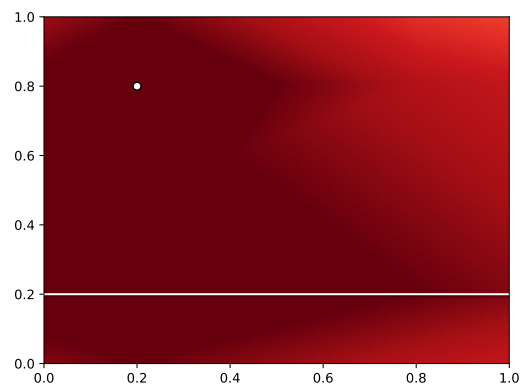

(a) $\bar{C}^{\max }$

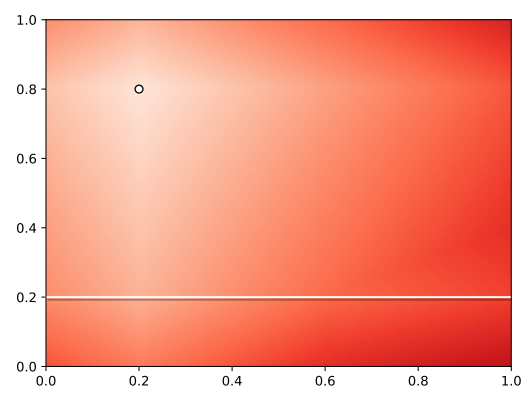

(c) $(X \backslash C)$

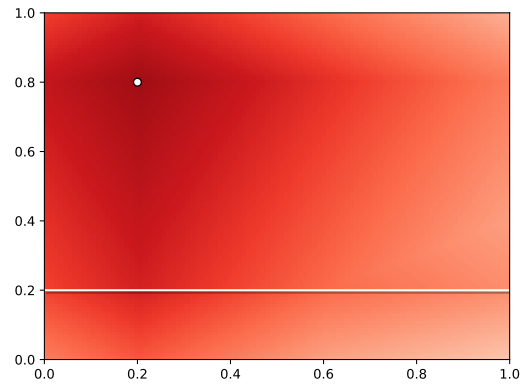

(b) $\bar{C}^{\text {min }}$

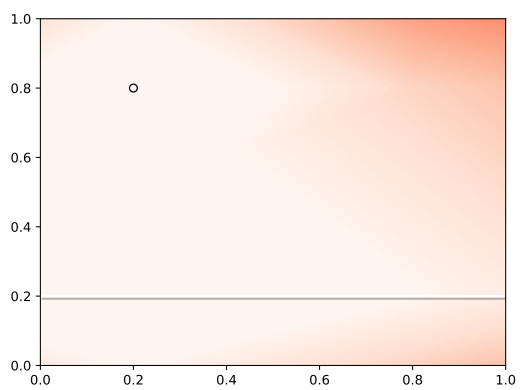

(d) $(X \backslash C)_{\min }$

Fig. 1: Toy example with $C=\{(0.2,0.8),(?, 0.2)\}$. Missing value displayed as a line. Optimistic and pessimistic upper approximations of $C$ and optimistic and pessimistic lower approximations of $X \backslash C$.

tolerance relation, we select $R_{i}(y, x)=1-\left|y_{i}-x_{i}\right| / r_{i}$, where $r_{i}$ is the range of values in the training set.

We evaluate performance with the mean Area Under the Receiver Operator Curve (AUROC) across 5-fold cross-validation. For multi-class datasets, we use the extension of AUROC by Hand \& Till (2001) [11]. We apply this to eleven datasets with missing values selected from the UCI archive of machine learning datasets [6]. Where applicable, we remove classes with fewer than five instances, and select a stratified sample of 2000 instances.

We experiment with two strategies: using the mean membership values in the optimistic and pessimistic approximations, and optimising a weighted mean on the basis of training data.

For the second strategy, we use the efficient form of leave-one-out validation detailed in [19]. Briefly, this entails taking a single nearest neighbour query for 
the entire training set, and correcting it by removing nearest neighbour distances from a training instance to itself. The remaining values can then be used to calculate optimistic and pessimistic approximation memberships $\overline{C \backslash\{x\}}^{\text {max }}(x)$ and $\overline{C \backslash\{x\}}{ }^{\text {min }}(x)$. We parametrise the average of these two values with a value $\lambda \in[0,1](5)$.

$$
(1-\lambda) \cdot \overline{C \backslash\{x\}}^{\text {min }}(x)+\lambda \cdot \overline{C \backslash\{x\}}^{\text {max }}(x)
$$

We optimise $\lambda$ by calculating the resulting AUROC and applying MalherbePowell optimisation $[15,16]$ with a budget of 20 evaluations.

Note that the computational complexity of this strategy is equal to the computational complexity of a $k+1$-nearest neighbour query with $n$ query instances and $n$ target instances, where $n$ is the size of the training set. For large $n$, this can potentially be mitigated by using only a subset of the training set to optimise $\lambda$.

Table 1: Datasets with the number of classes, instances and attributes, the rate of missing values, and the AUROC from classification with the mean of optimistic and pessimistic upper approximation memberships, with an optimised ratio of both, and with normal upper approximation memberships after imputation with the mean and mode.

\begin{tabular}{|c|c|c|c|c|c|c|}
\hline \multirow{2}{*}{$\begin{array}{l}\text { Dataset } \\
\text { adult }\end{array}$} & $c$ & \multirow{2}{*}{$\begin{array}{r}n \\
2000\end{array}$} & \multirow{2}{*}{$\begin{array}{l}m \quad \# ? \\
130.010\end{array}$} & \multicolumn{3}{|c|}{ Mean Optimised Imputation } \\
\hline & & & & 0.863 & 0.863 & 0.860 \\
\hline aps-failure & & 2000 & 1700.083 & 0.969 & 0.985 & 0.993 \\
\hline arrhythmia & 10 & 443 & 2790.003 & 0.878 & 0.880 & 0.877 \\
\hline ckd & 2 & 400 & 240.105 & 1.000 & 1.000 & 1.000 \\
\hline exasens & 4 & 399 & 70.428 & 0.738 & 0.748 & 0.734 \\
\hline hcc & 2 & 165 & 490.102 & 0.746 & 0.741 & 0.771 \\
\hline hepatitis & 2 & 155 & 190.057 & 0.879 & 0.884 & 0.877 \\
\hline mammographic-masses & 2 & 961 & 40.042 & 0.833 & 0.834 & 0.827 \\
\hline primary-tumor & 15 & 330 & 170.039 & 0.779 & 0.777 & 0.775 \\
\hline secom & & 15675 & 5900.045 & 0.678 & 0.681 & 0.689 \\
\hline soybean & 19 & 683 & 350.098 & 0.993 & 0.995 & 0.996 \\
\hline Mean & & & & 0.851 & 0.854 & 0.854 \\
\hline
\end{tabular}

The results are displayed in Table 1 . Optimising the weighted mean increases AUROC for 7 datasets and decreases it for 3. Applying a one-sided Wilcoxon signed-rank test, we find that this is weakly significant $(p=0.057)$.

For comparison, we have also included the results obtained from simple imputation with the mean (numerical attributes) or mode (categorical attributes) of the known values in the training data. For 6 datasets, both the mean and optimised weighted mean optimistic and pessimistic approximations achieve a higher AUROC than simple imputation, whereas for 5 datasets, simple impu- 


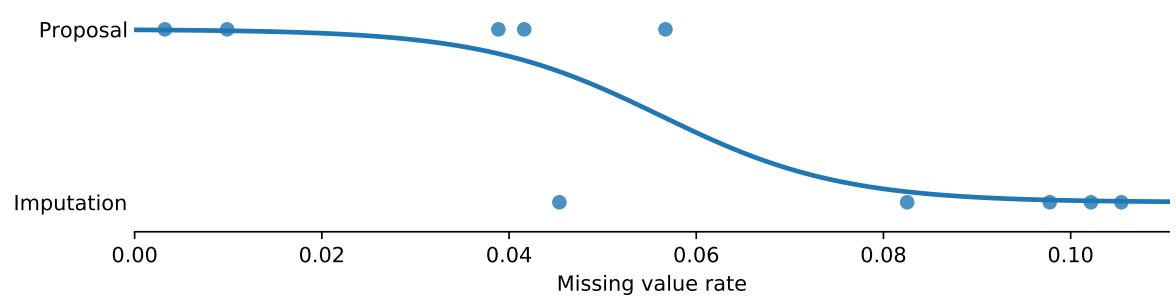

Fig. 2: Distribution of datasets for which imputation or the proposal of this paper achieves higher AUROC as a function of the missing value rate, with logistic regression fit.

tation achieves a higher AUROC. If we exclude the outlying dataset exasens, we see that the optimistic and pessimistic approximations perform better on datasets with a lower missing value rate, and imputation on datasets with a higher missing value rate (Fig. 2). When we fit a logistic regression model, the odds are even at a missing value rate of 0.056 .

\section{Conclusion}

In this paper we have presented an approach towards datasets with missing values that has received relatively little attention so far. While the existing literature is typically devoted to resolving these missing values in an optimal manner, we have argued that in the context of fuzzy rough sets, which are motivated by a desire to model different kinds of uncertainty, it is worthwhile to also model the uncertainty of incomplete information.

We have recalled the concept of interval-valued fuzzy rough set, which iterates on the dualistic nature of rough sets and replaces the upper and lower approximations by interval-valued fuzzy sets: secondary pairs of approximations, reflecting optimistic and pessimistic assumptions about the values that are missing. These define a bandwidth that contains the true (but unknown) upper and lower approximation memberships. We think that this can offer a valuable perspective for qualitative analyses of datasets with missing values.

We have shown how the interval-valued upper and lower approximations can be used to extend Fuzzy Rough Nearest Neighbour (FRNN) classification to problems with missing values. From an evaluation on several real-world datasets, we found that the best results can be obtained by taking a weighted average of the optimistic and pessimistic approximation memberships, and by optimising the relative weight on the basis of training data. This results in a comparable overall performance to simple imputation with the mean and mode, but is more directly interpretable as it does not involve the insertion of artificial values. Further analysis revealed that our proposal in particular outperforms imputation on datasets with a missing value rate below 0.056 . 
We leave the integration of interval-valued fuzzy rough sets into other algorithms like fuzzy rule induction for future research.

\section{Acknowledgement}

We would like to thank two anonymous reviewers for bringing the similar proposals [14] and [5] to our attention.

The research reported in this paper was conducted with the financial support of the Odysseus programme of the Research Foundation - Flanders (FWO). D. Peralta is a Postdoctoral Fellow of the Research Foundation - Flanders (FWO, 170303/12X1619N).

\section{References}

1. Amiri, M., Jensen, R.: Missing data imputation using fuzzy-rough methods. Neurocomputing 205, 152-164 (2016)

2. Aste, M., Boninsegna, M., Freno, A., Trentin, E.: Techniques for dealing with incomplete data: a tutorial and survey. Pattern Analysis and Applications 18(1), 1-29 (2015)

3. Bellman, R., Kalaba, R., Zadeh, L.: Abstraction and pattern classification. Tech. Rep. RM-4307-PR, The Rand Corporation, Santa Monica, California (1964)

4. Cornelis, C., Verbiest, N., Jensen, R.: Ordered weighted average based fuzzy rough sets. In: RSKT 2010: Proceedings of the 5th International Conference on Rough Set and Knowledge Technology. Lecture Notes in Artificial Intelligence, vol. 6401, pp. 78-85. Springer (2010)

5. Couso, I., Dubois, D.: Rough sets, coverings and incomplete information. Fundamenta Informaticae 108(3-4), 223-247 (2011)

6. Dua, D., Graff, C.: UCI machine learning repository (2019), http://archive.ics.uci. $\mathrm{edu} / \mathrm{ml}$

7. Dubois, D., Prade, H.: Twofold fuzzy sets and rough sets - some issues in knowledge representation. Fuzzy Sets and Systems 23(1), 3-18 (1987)

8. Dubois, D., Prade, H.: Rough fuzzy sets and fuzzy rough sets. International Journal of General Systems 17(2-3), 191-209 (1990)

9. Dubois, D., Prade, H.: Interval-valued fuzzy sets, possibility theory and imprecise probability. In: EUSFLAT-LFA 2005: Proceedings of the Joint 4th Conference of the European Society for Fuzzy Logic and Technology and the 11th Rencontres Francophones sur la Logique Floue et ses Applications. pp. 314-319 (2005)

10. Grzymala-Busse, J.W.: Rough set strategies to data with missing attribute values. In: Foundations and novel approaches in data mining, pp. 197-212. Springer (2006)

11. Hand, D.J., Till, R.J.: A simple generalisation of the area under the ROC curve for multiple class classification problems. Machine learning 45(2), 171-186 (2001)

12. Hong, T.P., Tseng, L.H., Chien, B.C.: Mining from incomplete quantitative data by fuzzy rough sets. Expert Systems with Applications 37(3), 2644-2653 (2010)

13. Jensen, R., Cornelis, C.: A new approach to fuzzy-rough nearest neighbour classification. In: RSCTC 2008: Proceedings of the 6th International Conference on Rough Sets and Current Trends in Computing. Lecture Notes in Artificial Intelligence, vol. 5306, pp. 310-319. Springer (2008) 
14. Jensen, R., Shen, Q.: Interval-valued fuzzy-rough feature selection in datasets with missing values. In: FUZZ-IEEE 2009: Proceedings of the 18th International Conference on Fuzzy Systems. pp. 610-615. IEEE (2009)

15. King, D.E.: Dlib-ml: A machine learning toolkit. Journal of Machine Learning Research 10(60), 1755-1758 (2009)

16. King, D.E.: A global optimization algorithm worth using. http://blog.dlib.net/ 2017/12/a-global-optimization-algorithm-worth.html (Dec 2017), last accessed 6 Jan 2021

17. Kryszkiewicz, M.: Rough set approach to incomplete information systems. Information sciences 112(1-4), 39-49 (1998)

18. Lenz, O.U., Peralta, D., Cornelis, C.: Scalable approximate FRNN-OWA classification. IEEE Transactions on Fuzzy Systems 28(5), 929-938 (2020)

19. Lenz, O.U., Peralta, D., Cornelis, C.: Optimised one-class classification performance (2021), https://arxiv.org/abs/2102.02618

20. Pawlak, Z.: Rough sets. Rep. 431, ICS PAS (1981)

21. Pawlak, Z.: Rough sets. International Journal of Computer \& Information Sciences 11(5), 341-356 (1982)

22. Thangavel, K., Pethalakshmi, A.: Dimensionality reduction based on rough set theory: A review. Applied Soft Computing 9(1), 1-12 (2009)

23. Zadeh, L.A.: Fuzzy sets. Information and Control 8(3), 338-353 (1965) 